\title{
Review
}

\section{Role of Toll-like Receptors in Adjuvant-Augmented Immune Therapies}

\author{
Tsukasa Seya $^{1,2}$, Takashi Akazawa ${ }^{2}$, Tadayuki Tsujita ${ }^{1,3}$ and Misako Matsumoto, ${ }^{1,2}$ \\ ${ }^{1}$ Department of Microbiology and Immunology, Hokkaido University Graduate School of Medicine, \\ Kita-15, Nishi-7, Kita-ku Sapporo 060-8638 and ${ }^{2}$ Department of Immunogy, Osaka Medical Center for Cancer, \\ Nakamichi 1-3-2, Higashinari-ku, Osaka 537-8511, Japan
}

\begin{abstract}
Effective therapeutic vaccines contain two primary constituents, antigen and adjuvant. Adjuvants consisting of microbial pattern molecules play a central role in vaccination. Successful vaccine requires efficient induction of antibody (Ab), type I interferons (IFN), cytokines/chemokines, cytotoxic $\mathrm{T}$ lymphocytes (CTL) and/or NK cells. Toll-like receptors (TLRs) in myeloid dendritic cells (mDC) essentially act as adjuvant receptors and sustain the molecular basis of adjuvant activity. Current consensus is that TLRs and their adapters introduce signals to preferentially induce IFN- $\alpha / \beta$, chemokines and proinflammatory cytokines, and mature $\mathrm{mDC}$ to augment antigen presentation. Although most of these data were obtained with mice, the results are presumed to be adaptable to humans. Whenever TLR pathway is activated in $\mathrm{mDC}$, NK and/or CTL activation is promoted. For induction of antigenspecific CTL toward phagocytosed material, cross-priming must be induced in $\mathrm{mDC}$, which is also sustained by TLR signaling in $\mathrm{mDC}$. Since the TLR responses vary with different adjuvants, $\mathrm{mDC}$ functions are skewed depending on adjuvant-specific direction of $\mathrm{mDC}$ maturation. It appears that the directed maturation of $\mathrm{mDC}$ largely relies on selection of appropriate sets of TLRs and their adapter signaling pathways. Synthetic chimera molecules consisting of TLR agonists and target antigens are found to be effective in induction of CTL to eliminate target cells in vivo. Here, we review the role of human TLRs and adapters in a variety of host immune responses. We will also describe the relevance of adjuvants in the manipulation of receptors and adapters in vaccine therapy.
\end{abstract}

Keywords: adjuvants - dendritic cells - interferon - microbial pattern molecules - Toll-like receptor

\section{Adjuvants as ligands for TLRs}

Microbial pattern molecules with a high potential for host immune activation have been classified into adjuvant. Adjuvant activity has been found in a variety of infectious microbes and endogenous material of host origin. Freund's complete adjuvant (FCA) consisting of dead mycobacteria conjugated with mineral oil augments vaccine response, i.e. antibody

${ }^{3}$ Present address: ERATO-JST, Laboratory of Molecular and Developmental Biology, University of Tsukuba, Tennoudai 1-1-1, Tsukuba 305-8577, Japan

For reprints and all correspondence: Tsukasa Seya, Department of

Microbiology and Immunology, Graduate School of Medicine, Hokkaido

University, Kita-ku, Sapporo 060-8638 Japan. Tel: +81-11-706-5073;

Fax: +81-11-706-7866; E-mail: seya-tu@med.hokudai.ac.jp
(Ab) production, CTL induction and NK activation $(1,2)$. Without the adjuvant, usually only a poor immune response is observed upon vaccination. Thus, the adjuvant has been an essential factor for provoking strong host immune responses. It has been elucidated that myeloid dendritic cells (mDCs), a representative cell population of antigen-presenting cells, and plasmacytoid dendritic cells (pDCs), formerly called type I interferon (IFN)-producing cells, are the targets for most of the adjuvants (3). In this context, Janeway and Medzhitov (4) suggested that dendritic cells (DCs) express two sorts of receptors, antigen $(\mathrm{Ag})$ uptake receptors for $\mathrm{Ag}$-presentation by MHC and receptors for microbial pattern molecules, that are receptors for adjuvants. Both Ags and adjuvant pattern molecules are derived from microbes and differentially act

(C) The Author (2006). Published by Oxford University Press. All rights reserved.

The online version of this article has been published under an open access model. Users are entitled to use, reproduce, disseminate, or display the open access version of this article for non-commercial purposes provided that: the original authorship is properly and fully attributed; the Journal and Oxford University Press are attributed as the original place of publication with the correct citation details given; if an article is subsequently reproduced or disseminated not in its entirety but only in part or as a derivative work this must be clearly indicated. For commercial re-use, please contact journals.permissions@oxfordjournals.org 
on mDCs to efficiently induce many effectors secondary to lymphocyte activation.

The molecular basis of the adjuvant function was poorly delineated until adjuvant receptors were identified. The most common question in this regard is 'what is the receptor for adjuvant pattern molecules?' In 1997, Medzhitov et al. (5) characterized a human homolog of drosophila Toll (described by J. A. Hoffmann in 1996) (6), later named TLR4. They first predicted its function to be the augmentation of immune response $(4,5)$. Currently, TLR is identified as a family of receptors consisting of $>10$ protein members both in humans and mice. In recent years, evidence has been accumulating that either each TLR dimer or a combination of TLRs serves as a receptor complex for the recognition of a specific microbial pattern molecule $(3,7)$. The recognition is then followed by TLR-specific signaling and corresponding cellular immune response (Table 1). Thus, the TLR family of proteins serves as signaling receptors crucial for augmenting immune response. It is notable that most of the TLR proteins reside in DCs. Although some TLRs are present on T, B, NK cells and epithelial cells and recent studies suggest the importance of TLR on these cells for primary antiviral response (8), their functions remain largely unknown. The possible immune responses and effectors induced by $\mathrm{mDC}$ are discussed below.

\section{Role of TLRS in DCs}

mDCs are central to T/B cell activation $(9,10)$. They facilitate production of Abs through the induction of differentiation of B lymphocytes. T lymphocytes are differentiated by matured mDCs into T helper I (Th1), Th2 and CTL. mDCs catch up antigens, alter the functions and migrate to draining lymphnodes (Fig. 1). When antigen and adjuvancy coincidently stimulate $\mathrm{mDC}$ maturity, antigenic peptide presentation on
MHC is augmented. Upregulation of co-stimulatory and MHC molecules, expression of chemokine receptors, presentation of antigens, secretion of cytokines and chemokines are accelerated by adjuvants in $\mathrm{mDC}(7,9,10)$. TLRs on mDCs are involved in these pivotal events leading to lymphocyte activation (Fig. 1). Regulatory $\mathrm{T}$ cell (Treg) function is suppressed by IL-6, which is produced by $\mathrm{mDC}$ in response to TLR activation (11). NK and NKT cells are activated in response to TLR-mediated $\mathrm{mDC}$ maturation (12). Memory cell formation may be imparted by $\mathrm{mDC}$ depending on its maturation properties, which are competent to lymphocyte

Table 1. Human TLRs and their adapters

\begin{tabular}{lclllll}
\hline huTLR & $\begin{array}{l}\text { Amino } \\
\text { acids }\end{array}$ & mAb & Adapters & Ligands & $\begin{array}{l}\text { DC } \\
\text { subsets }\end{array}$ & Modes \\
\hline TLR1 & 786 & TLR1.136 & M-1/M-2 & triacyl BLP & M & NF-kB \\
TLR2 & 784 & TLR2.45 & M-1/M-2 & PGN, BLP & M & NF-kB \\
TLR3 & 904 & TLR3.7 & T-1 (M-1) & dsRNA & M & NF-kB/IRF-3 \\
TLR4 & 839 & HTA125 & M-1/M-2 & LPS, Taxol & M & NF-kB/IRF-3 \\
& RSV-F & & & & & \\
TLR5 & 858 & - & M-1 & flagellin & M & NF-kB \\
TLR6 & 796 & TLR6.127 & M-1/M-2 & diacyl BLP & M & NF-kB \\
TLR7 & 1049 & TLR7.360 & M-1 & ssRNA & P & NF-kB/IRF-7 \\
TLR8 & 1059 & TLR8.90 & M-1? & ssRNA & M & NF-kB/IRF-7 \\
TLR9 & 1032 & - & M-1 & CpG DNA & P & NF-kB/IRF-7 \\
\hline
\end{tabular}

M-1, MyD88; M-2, Mal/TIRAP; T-1, TICAM-1/TRIF; T-2, TICAM-2/ TRAM. TLR1, TLR6 and TLR10 are members of the TLR2 subfamily and together with TLR2 recognize different sets of microbial pattern molecules and support activation of TLR2. Mice do not have TLR8, but possess a protein which resembles TLR7 in its structure and function. Further, their distribution is also different. Functional modes of each adapter were identified as ' $M$ ' and ' $\mathrm{T}$ ' types. M, MyD88-dependent pathway; T, TICAM-1-dependent pathway. In DCs subsets, $\mathrm{M}$ is myeloid DCs while $\mathrm{P}$ is plasmacytoid DCs. 'Modes' represent transcription factors activated by each TLR.

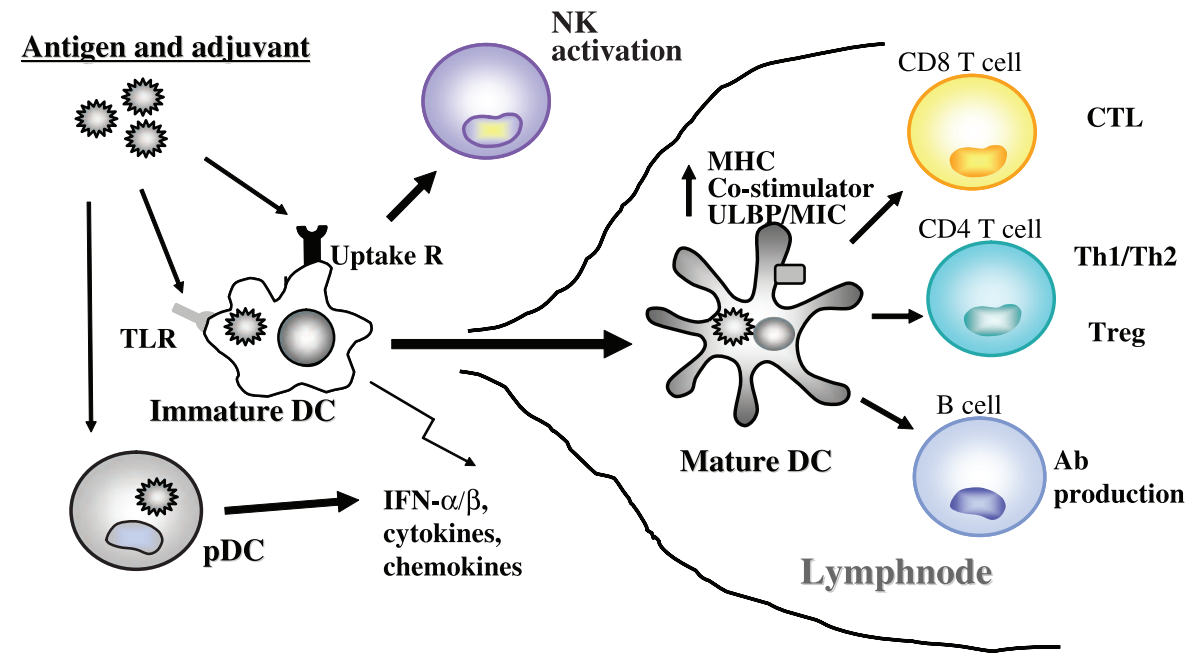

Figure 1. Role of human TLRs in $\mathrm{mDC}$ maturation followed by activation of various lymphocytes. Immature dendritic cells (mDC) residing in local tissue phagocytose exogenous antigen $(\mathrm{Ag})$ and pattern molecule (namely adjuant) and initiate the maturation process. During maturation mDC induce IFNs, cytokines and chemokines, allow the upregulation of co-stimulators, NK-activating ligands (ULBP, MIC, etc) and MHC, and activate a variety of lymphocytes. These maturation events are largely dependent on adjuvant properties. Also, adjuvant may participate in switching on of some unknown mechanisms which are essential in induction of CD8+ CTL by mDCs. 
proliferation, and be established in conjunction with TLR signaling in mDCs. Exogenously-added antigens that are not expected to gain access to the cytoplasm of mDCs are presented on MHC class I molecules by a process called crosspriming. TLR signaling also promotes cross-priming in $\mathrm{mDC}$, which potently induces MHC class I-restricted CTL against phagocytosed antigens (13). This TLR-driven crosspriming will be discussed more in the later sections. Simultaneous stimulation of DC by microbial patterns and antigens induces robust immune activation. It is notable that DC subsets have distinct receptor-expression profiles which enable them to respond to specific types of pathogens $(7,14)$. In addition, mDCs express pattern-recognition receptors other than TLRs inside the cells (15). Furthermore, there are a number of microbe-specific phagocytic receptors in $\mathrm{mDC}$, which include lectins, Ig superfamily and complement receptors. Functional assignment of TLRs and these intracellular and phagocytic receptors in $\mathrm{mDC}$ are important to depict the DC pattern recognition system. Elucidation of the kind of TLRs and their combinations that are mainly responsible for each immune response is to be further resolved.

\section{The distribution of TLR expression in DC subsets}

TLR1, 2, 4, 5 and 6 are members of a TLR subfamily which recognizes microbial constituents that are absent in human cells (Table 1). These TLR subfamily members reside on the cell surface. Human mDCs, but not pDCs express these TLR subfamily members (14). Human mDCs also express TLR3 and TLR8 while pDCs express TLR7 and TLR9. These four TLRs preferentially recognize microbe-specific modifications of nucleotide sequences and are localized in certain endosomes inside the cells $(14,16)$. Unlike mouse mDCs, human mDCs do not express TLR9. Current consensus is that TLR3, 7,8 and 9 are proteins of TLR subfamily participating in the recognition of nucleic acid derivatives of viruses and bacteria (Table 1). In mice, mDCs express functional TLR3 and TLR9 inside the cell, while TLR8 remains dysfunctional (16). Mouse pDC expresses TLR7 and TLR9 similar to human. These differences between mouse and human DC subsets may preclude us from simple extrapolation of mouse TLR functions in mouse DCs to the human system. Typical human pDC phenotype is $\mathrm{CD} 11 \mathrm{C}^{-} / \mathrm{CD}^{+}{ }^{+}$with $\mathrm{BDCA} 4+$, while that

Table 2. Distribution and subcellular localization of human TLRs in DC

\begin{tabular}{|c|c|c|c|c|c|c|c|c|c|}
\hline \multirow[t]{2}{*}{ DC subsets } & \multicolumn{9}{|c|}{ Monoclonal Abs against } \\
\hline & TLR1 & TLR2 & TLR6 & TLR4 & TLR5 & TLR3 & TLR7 & TLR8 & TLR9 \\
\hline $\begin{array}{l}\text { Monocyte- } \\
\text { derived }\end{array}$ & + & ++ & + & + & + & $(++)$ & - & $(++)$ & - \\
\hline Plasmacytoid & - & - & - & - & - & - & $(++)$ & - & $(++)$ \\
\hline
\end{tabular}

$(++)$ TLRs with nucleotide-recognition properties (TLR3, 7, 8. 9) reside in endosomes.

+ TLRs (TLR1, 2, 4, 5, 6) are expressed on cell surface to recognize microbial patterns.

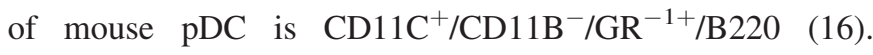
Thus, mouse and human also differ in the pDC phenotype. Since we mainly focus on antigen-presenting ability of $\mathrm{mDC}$ in the context with TLR adjuvancy, the pDC story (see other references) is largely omitted in this review.

\section{Functions of the adapters}

So far, four adaptors, MyD88, Mal/TIRAP, TICAM-1 (TRIF) and TICAM-2 (TRAM), have been identified in human and mouse cells (17-19). These adapters exclusively contain the TIR domain (Fig. 3). We have investigated the functions of adapters and found that stimulation of $\mathrm{mDC}$ with individual pattern molecules is largely transduced by different sets of adapters resulting in distinct outputs. TLR subfamily expressed on cell surface mainly engages in NF- $\kappa \mathrm{B}$ activation through the adapter molecule MyD88 (17) (Fig. 2). TLR4 is the only exception in that it activates both NF- $\mathrm{KB}$ and IRF-3. In contrast, the nucleic acid-recognizing TLRs, TLR3, 7 and 9 activate the IFN promoter (Fig. 2). The properties of these adapters related to the activation of $\mathrm{mDC}$ (i.e. antigenpresenting cell) may be summarized as follows: MyD88 is engaged in the activation of NF- $\kappa \mathrm{B}$ and p38 MAPK (17), but has no ability to induce type 1 IFN in mDCs. An alternatively spliced form of MyD88 may regulate the MyD88 adapter activity. Mal/TIRAP bridges the TIR domain of TLR2/4 and MyD88 (18,19). Mal/TIRAP itself exhibits weak NF- $\kappa \mathrm{B}$ activation activity. The Mal/TIRAP function may be modulated by proteolysis.

TICAM-1 preferentially activates the IFN- $\beta$ promoter via dimerization of IRF-3 in mDC (20), which explains part of the 'MyD88-independent' pathway. TICAM-1 has ability to activate NF- $\kappa \mathrm{B}$ also. The TICAM-1-mediated NF- $\kappa \mathrm{B}$ activation is supported by RIP1 which binds the C-terminus of TICAM-1 (21). However, TICAM-1-dependent IRF-3 activation is made by the NAP1/TBK1/IKK $\epsilon$ complex (22) that binds to the N-terminus of TICAM-1. Particularly if it is of viral, TICAM-1 may be susceptible to proteases (23). TICAM-2 bridges the TIR domain of TLR4 and TICAM-1 $(18,19)$. Its ability to activate IRF-3 in the absence of TICAM-1 is minimal, if any. Its acylation permits the molecule to anchor on the inner leaflet of membrane. Hence, the two effective adaptors, MyD88 and TICAM-1, and two bridging adaptors, Mal/TIRAP and TICAM-2 exist in DCs (Fig. 3).

TLR3 activates IRF-3 and IFN- $\beta$ promoter through the adapter TICAM-1, whereas TLR7 and 9 activate IRF-7 and IFN- $\alpha$ promoter via MyD88 (24). The distribution of these TLRs is different: TLR3, TLR8 and surface-expressed TLRs reside in antigen-presenting $\mathrm{mDCs}$ while TLR7 and 9 are in pDCs in human (Table 2). Thus, the TLR3, TLR8 and surface-expressed TLRs such as TLR2, 4 and 5 are mainly involved in the modulation of antigen-presentation in $\mathrm{mDC}$ (Fig. 3).

The functional profile of TLR2 and TLR5 includes activation of NF- $\mathrm{KB}$ (17) but not induction IFN- $\beta$, which matches the functional properties of the adapters they select. 


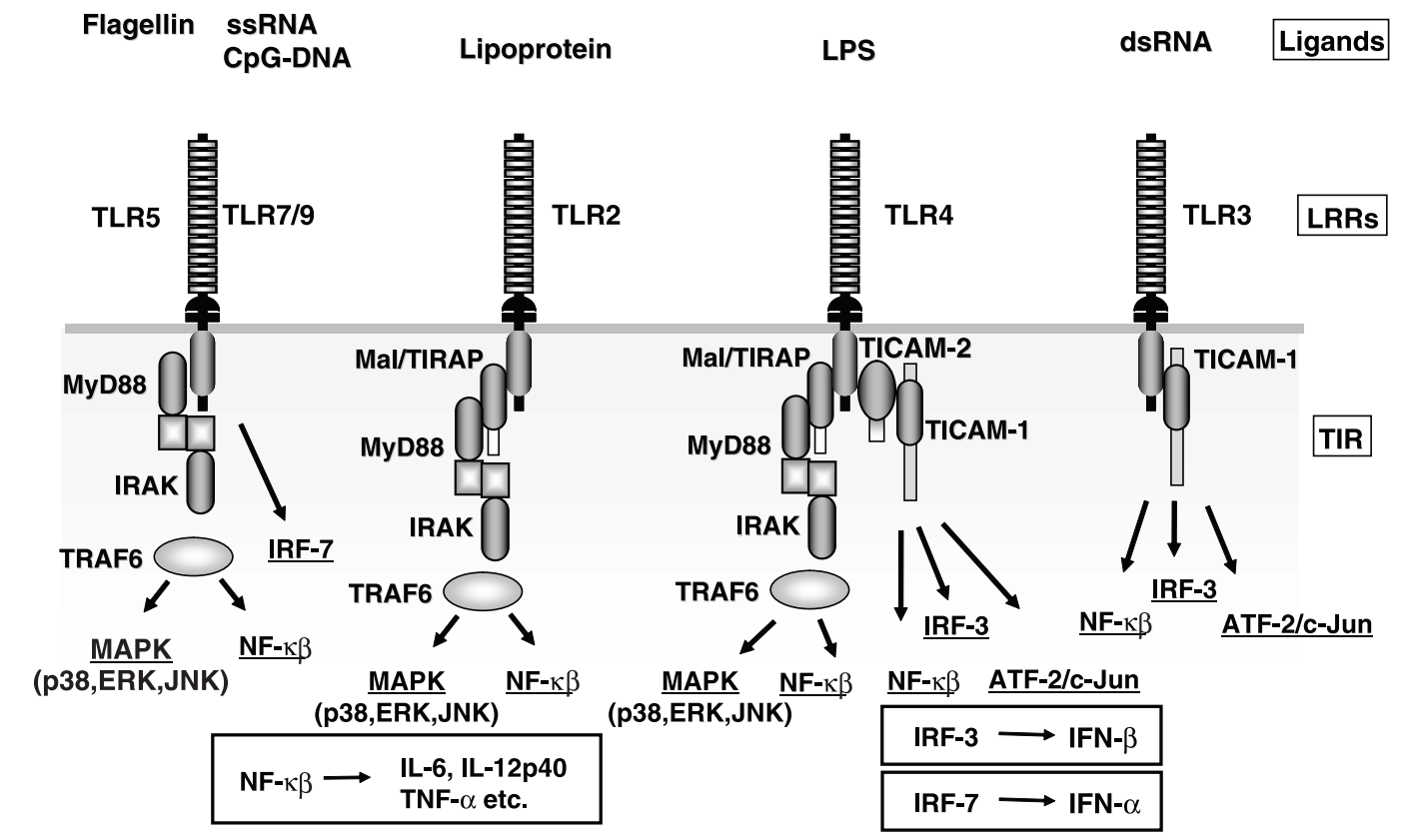

Figure 2. Association between human TLRs and adaptors determine each TLR-specific signaling pathway. Topology of the adaptor proteins in the TIR domains of TLR2, TLR3 and TLR4 is shown in the schema. The complex consisting of each TIR and adaptors delivers TLR signaling to activate NF- $\mathrm{KB}$ and the IFN- $\beta$ promoter (IRF-3). In pDC, activation of TLR7 or 9 happens to activate IRF-7 in a MyD88-dependent way followed by induction of IFN- $\alpha$. Representative ligands of TLRs are shown on the top.

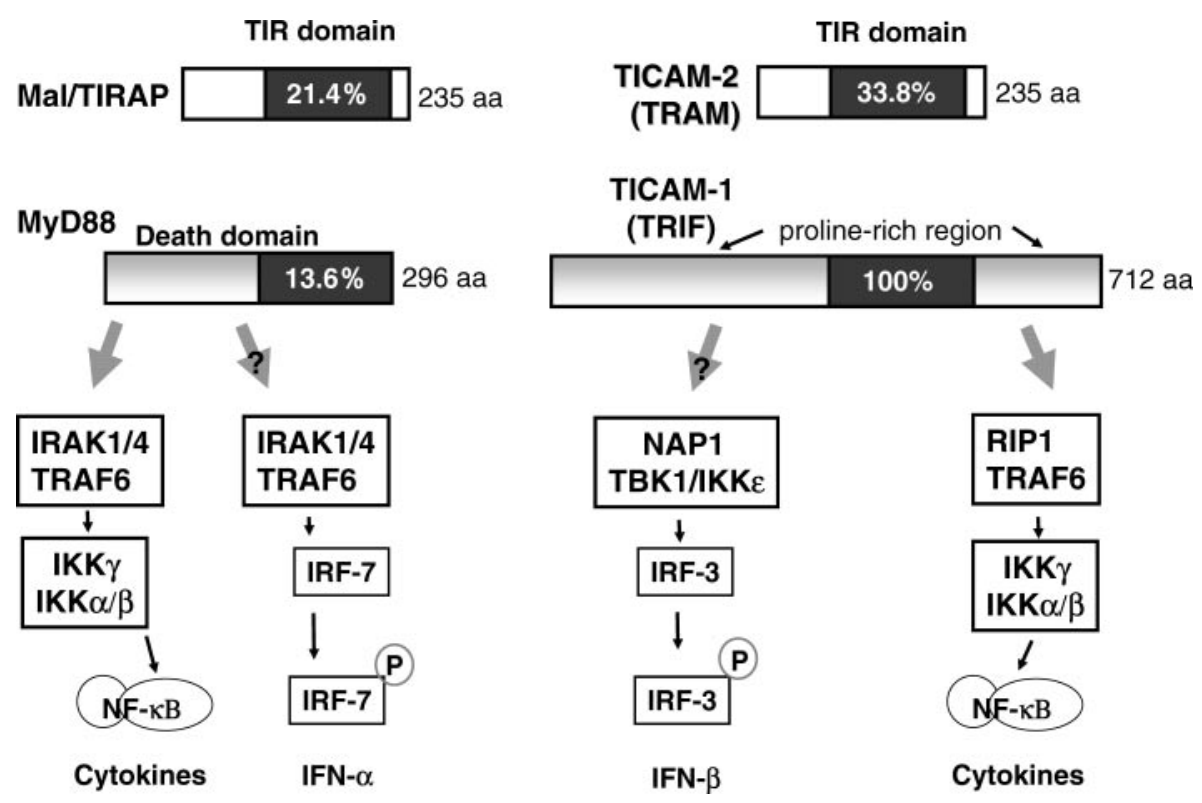

Figure 3. TLR adapters and possible signals induced in human DCs. MyD88 and TICAM-1 are functional adapters that activate both NF- $\kappa B$ and type I IFN promoters. Important downstream molecules currently identified are shown in the figure. Plasmacytoid DC (pDC) predominantly expresses TLR7 and TLR9 which recognize nucleotide derivatives. MyD88 activates IRF-7 in pDCs. Activation of different pathways is elicited in mDC.

dsRNA-mediated TLR3 activation predominantly induces IFN- $\beta$, reflecting the function of the adapter TICAM-1 $(18,19)$. LPS-TLR4 complex activates both NF- $\kappa \mathrm{B}$ and the IFN- $\beta$ promoter, consistent with the fact that TLR4 recruits both MyD88 and TICAM-1 in an indirect manner. Thus, sets of adapters selected by each TLR appear to be crucial for relevant adjuvant function.

\section{Manipulation of TLR system for adjuvant vaccine therapy}

Functions of antigen-presenting $\mathrm{mDC}$ can be controlled at three points in the TLR cascade. Property of the adjuvant is the first to regulate TLR-mediated maturation of $\mathrm{mDC}$. Secondly, modulation of TLR activity occurs by the addition 
of soluble forms of TLRs or TLR-blocking molecules. Finally, TLR signaling can be positively regulated by the adapters or negatively regulated by their inhibitors. Recent finding suggests that there are small synthetic molecules that block TLR-adapter interaction (25). More information about TLR agonists in association with adjuvants was recently published (26). Examples for the second and third control steps are as follows.

Alternatively spliced forms of TLR2, TLR4 and probably TLR3 appear to serve as dominant-negatives to block the relevant TLR activation in response to their ligands (27-29). Reports on this type of TLR regulation were published in human and mouse TLRs (27-29). Fish has a gene encoding a soluble TLR5, which acts as an amplifier of the flagellinmediated membrane TLR5 signaling that induces acute phase cytokines as well as soluble TLR5 (30). Although human has no gene for soluble TLR5, the amplification of flagellin signal by soluble TLR5 may be conserved. Fish soluble TLR5 can physiologically bind flagellin and augment the functions of human membrane TLR5 in response to flagellin. The results may offer an adjuvant positively regulating flagellin response in human mDCs (31). Lipopolysaccharidebinding protein or soluble CD14 may upregulate LPSmediated TLR4 activation (32). It is likely that yet to be identified catch-up receptors for pattern molecules function as TLR modulators. Antagonists of TLRs either binding to the LRR or TIR domains of each TLR will be applicable for patients with inflammation or autoimmune status to block off TLR activity.

In the human kidney cell line HEK293 cells, overexpressed adapters enhance activation of corresponding pathways leading to the promoter activation even in the absence of adjuvant (22). Similarly, overexpressd dominant-negative forms of adapters effectively block the downstream signaling of adapters (22). In mDCs, adapter function can be positively regulated by the transfection of correspondingly associated adapters.

\section{Cross-priming induced by TLR stimuli in MDC}

TLRs and other receptors in $\mathrm{mDC}$ increase antigen-presenting ability by maturing mDC. However, the molecular mechanism whereby the TLRs and adaptors are potentially involved in antigen-presentation by $\mathrm{mDC}$ has not yet been characterized. Cross-priming is an essential functional feature for vaccines to induce CTL from CD8 T cells. Here, we mention a crucial function of TLR, cross-priming, in terms of TLR2/4 and TLR3. Although human TLR8 in $\mathrm{mDC}$ may participate in cross-priming, it remains unestablished. Effective CTL induction is observed secondary to TLR2/4 and MyD88-dependent $\mathrm{mDC}$ maturation if appropriate exogenous $\mathrm{Ag}$ is simultaneously supplemented in a study that used gene-knockout mice (33). Acylated devivatives of muramyl dipeptide (MDP) of BCG-cell wall skeleton (CWS) acts as TLR2/4 agonist to selectively activate the MyD88 pathway (34). Cytokines and chemokines are effectively induced in mDCs by combined activation of TLR2/4 by BCG-CWS leading to cross-primingcompetent mDC followed by CTL induction (33). The CTL induction by BCG-CWS-primed mDCs does not occur in MyD88-knockout mice and mDCs from them exerted almost no ability to proliferate CD8 T cells in vitro. Thus, it appears that there is a TLR-signal pathway which engages MyD88 to induce cross-priming in $\mathrm{mDC}$. The outline of this pathway is shown in Fig. 4 and more precisely in Fig. 5. Some unknown molecules involved in this pathway may participate in crosspriming of exogenously-added antigen in $\mathrm{mDC}$.

dsRNA is a TLR3 agonist that activates TLR3-TICAM-1 pathway (35). The outline of this pathway is shown in Fig. 4 and with molecular interaction in Fig. 5. TLR3 signaling is also important in eliciting cross-priming leading to induction of MHC class I presentation and CTL induction in response to exogenously-added antigens (36). In light of this observation, multiple TLR pathways must be involved in crosspriming in mDCs. These events may be generalized for most human infections and cancers where mDCs sense TLR agonists and antigens from target cells. Taken together, to induce systemic activation of CTL, supplementation of TLR agonists or adapters to the vaccine adjuvant appears effective, particularly if pathogens lack agonistic activity to TLR2/4 or TLR3.

\section{Vaccine adjuvant activity of TLR for NK activation}

NK can be activated by type 1 IFNs and/or instructive cytokines, IL-12, IL-23 and IL-18 (Fig. 1) (12). TLR3 engages in NK activation via TICAM-1-mediated signaling in $\mathrm{mDCs}$ (2). Thus, NK can be activated by mDCs that are pretreated with TLR3 agonist such as dsRNA. This means that mDC turns an activator for NK if appropriate TLR agonists are provided (Fig. 4). In light of this, it appears that supplementing vaccine adjuvant with TLR3 to induce systemic activation of NK appears to be effective in patients with cancer or infectious diseases.

If one can supplement the adjuvant cocktail sufficiently to activate both CTL and NK, it would be possible to eliminate both MHC-positive and -negative target cells. Although such a scenario is largely extended from the mouse studies, the same mechanism of induction of antiviral immunity appears to be the case in humans according to in vitro studies using human mDC $(7,8,14)$. Viral infection often induces promotion or suppression of DC maturation. Supplement of TLR2/4 agonists to viral dsRNA may relieve the maturation stages of DCs.

MyD88 is the adapter shared by receptors for IL-1 $\beta$, IL-18 and most members of the TLR family (17). In mDCs, the transcription factor $\mathrm{NF}-\kappa \mathrm{B}$ is activated in the MyD88 pathway (17). MyD88 may support events other than those responsible for innate immune responses and the danger signal induced by tumors $(37,38)$ or virus-invaded tissue in effecter lymphocytes $(39,40)$. In pDCs, the MyD88 pathway also activates IRF-7 which is followed by robust production of IFN- $\alpha$ (41). Type I IFN directly enhances the expression of IL-18 R 

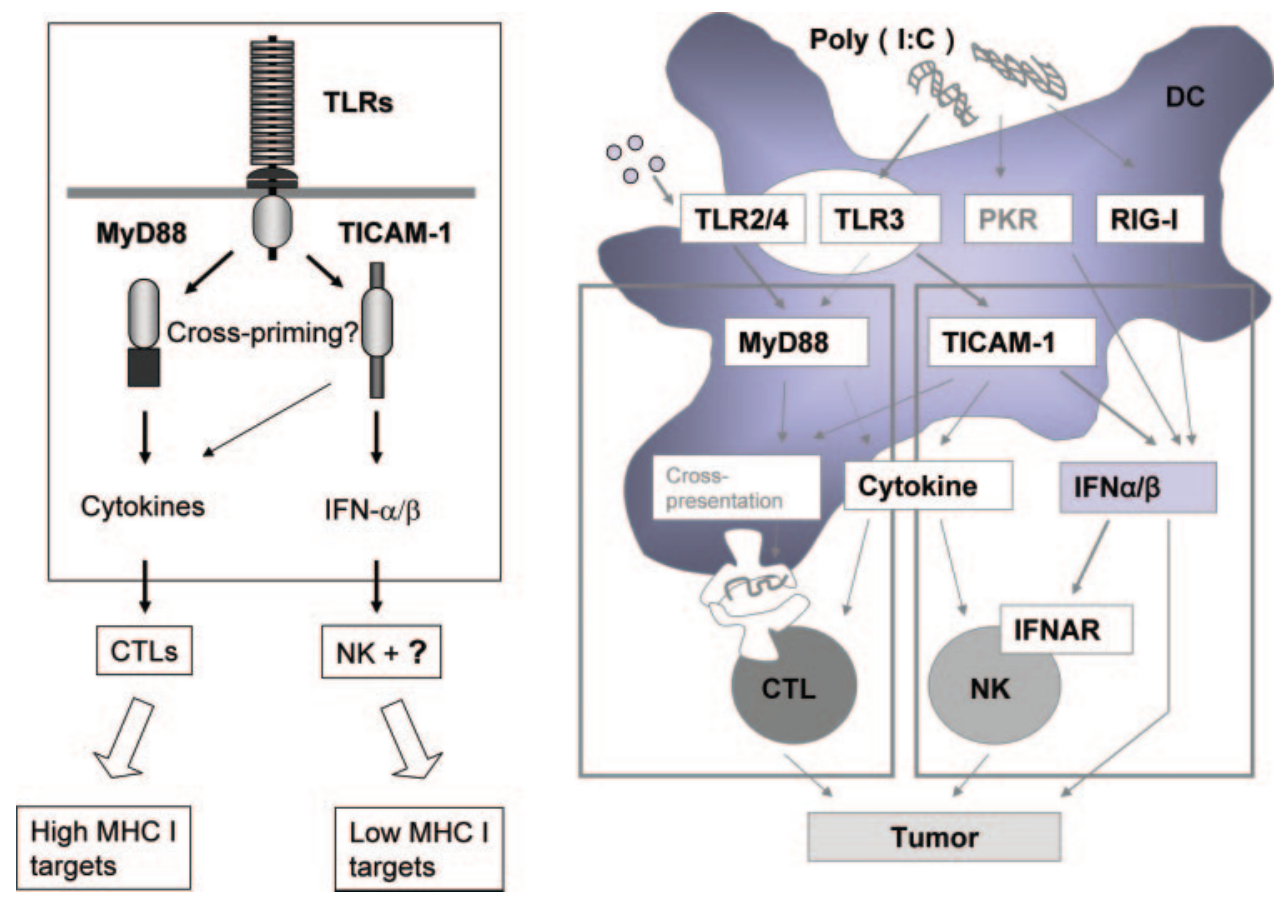

Figure 4. The two distinct TLR pathways of myeloid DCs. NK and CTL are induced as effecter cells for killing of targets secondary to activation of TLRs in mDCs (left panel). Myeloid DCs express TLR2, TLR3 and TLR4, and mature in response to BCG-CWS (TLR2/4 agonist) or dsRNA (TLR3 agonist). In general, TLR2/4 preferentially activates NF- $\mathrm{KB}$ via MyD88. TLR3 induces type 1 interferon (IFN). Both CTL and NK are then activated as indicated. In activation of CTL by mDC, exogenously-added antigen must be presented on MHC class I while the antigen are usually mounts on class II. This, named cross-priming, actually occurs if TLR signaling simultaneously enters (right panel). Either MyD88 or TICAM-1-mediated signal can induce cross-priming. Although the exact mechanism remains unknown, many factors are expected to be involved in switch on of cross-priming.

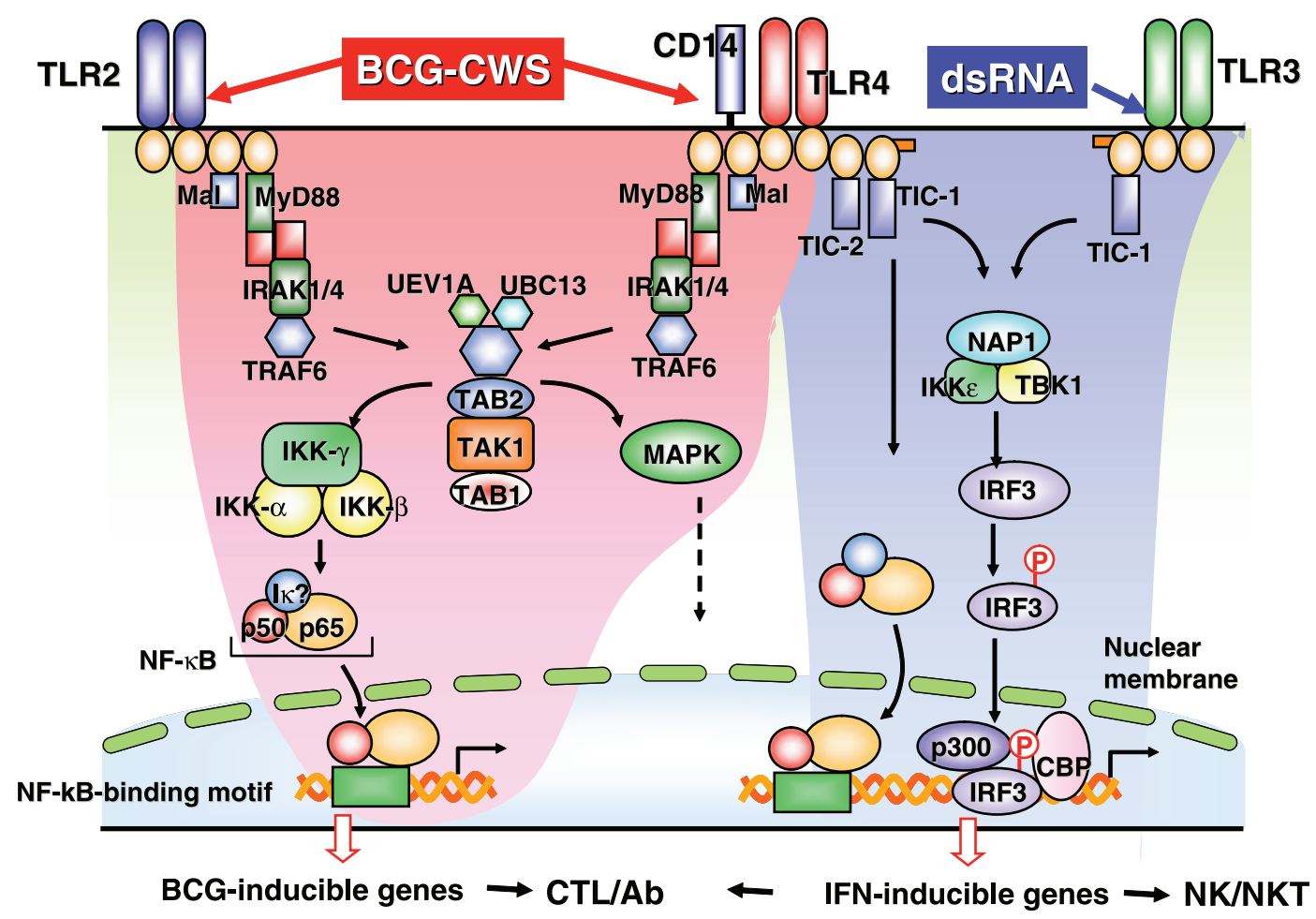

Figure 5. The two signaling pathways of TLR in myeloid DCs. MyD88-dependent pathway are indicated to the left and TICAM-1 pathway are shown to the right in blue. Many signaling molecules are involved in the two pathways leading to different outputs. NK and CTL are generated by the resultant mDC activation. In particular, cross-priming must occur in $\mathrm{mDC}$ to induce CTL, the molecular mechanism of which are largely unknown. 
components (AcPL), IL-1R-related protein (IL-1Rrp) and MyD88 in NK and T cells (39). This is reminiscent of the properties of the danger signal in the suppression of tumor cell progression or viral proliferation (42). It is reasonable to hypothesize that most danger signals suggest an enhanced effect of vaccine and activation of adapters. TICAM-1, another effective adapter, also induces activation of IFN- $\beta$ and NF- $\kappa B$ in mDCs $(18,19)$. It does not appear to function in pDCs for IFN- $\alpha$ induction. TICAM-1 may be an important vaccine potentiator targeting mDCs. Further studies are needed to clarify the discerning properties of the two main adapters in terms of enhancers for vaccine effect.

\section{Application of TLR response for immune therapy}

The development of safe and efficient vaccines for cancer and infectious diseases remains a major goal in global public health (43). There are prophylactic and therapeutic vaccines both of which require two primary constituents, antigen and adjuvant. Adjuvant may determine the direction of mDC leading to maturation for CTL induction and/or NK activation for cellular immune response and $\mathrm{Ab}$ production for humoral immune response. NK activation is a therapeutic feature, while CTL and Ab induction is useful for both preventive and therapeutic purpose.

Adjuvant includes a variety of components consisting of mineral oils, bacterial extracts, viruses and suspensions of aluminum hydroxide metals. Artificial components were chemically synthesized with reference to microbial origin, which were also effective as adjuvants $(26,44)$. In vitro and animal studies are in progress concerning these materials. Application of these adjuvants to immune therapies, however, has not yet clinically explored. This is because augmented immune response by adjuvants may exacerbate subclinical autoimmune diseases. In addition, fever and erosion at the local injected lesion induced by nature of adjuvants sometimes perturb periodical adjuvant administration in patients (45). Thus, the numbers of adjuvants that are approved and effective in humans still remain limited.

In the field of immune therapy for cancer, Rosenberg et al. (46) summarized their elaborate studies on peptide vaccine therapy for cancer. Only $2.6 \%$ of the patients with this therapy accomplish remission (complete or incomplete). In contrast, BCG or its component cell wall skeleton has been administered as adjuvant to patients with colon or lung cancer $(47,48)$. About $10 \%$ of the patient obtained partial remission and 5 year survival $(45,47)$. The results indicated that there is no doubt that the sensing of 'danger' by the immune system through recognition of microbial patterns by TLRs and other receptors has begun to explain the largely empirical field of vaccine adjuvant biology. It has also spawned a more objective search and development of compounds with immune stimulating activity (49). Our perspective is that combination of peptide and adjuvant would be more effective than either alone for immune therapy for cancer.
In this context, we have investigated the differential functional properties of each adjuvant in vitro human $\mathrm{mDC}$ and in vivo mouse studies (40). We particularly focused on microbial pattern molecules and TLR signal modifiers. We have examined the use of chimera proteins composed of small lipopeptides (TLR2 stimulants MALP-2 and Pam 3) and tumor antigens and found that their potencies to elicit immune response are greater than those of either alone or unfused mixture. It has been reported that fused proteins consisting of TLR agonists and targeted antigens exerts antipathogenic CTL response in vivo in animal studies $(50,51)$. Thus, we have speculated that fusion molecules consisting of TLR agonists and tumor antigens would be more effective for immune therapy for cancer.

TLRs are undoubtedly important in recognition of 'danger' molecules generated from self or infectious materials (53). TLRs are representative adjuvant receptors with sufficient knowledge on molecular manipulation and control of their signaling pathways. This is why we summarized this here. Yet, whether any of the numerous alternative and complementary medicines that claim immune activity contain TLR agonists and promote anti-pathogen immunity or autoimmunity remains to be established. Further future work on not only TLRs but also other adjuvant receptors will be needed to offer each of the adjuvants with differential properties for clinical use.

\section{Acknowledgements}

We are grateful to Drs K. Toyoshima and I. Azuma for organizing our project. Thanks are also due to Drs K. Funami, M. Shingai, A. Ishii, M. Sasai and A. Matsuo of our laboratory for their critical discussions. This work was supported in part by CREST, JST (Japan Science and Technology Corporation), and by Grants-in-Aid from the Ministry of Education, Science, and Culture (Specified Project for Advanced Research), the Ministry of Health and Welfare (Organization for Pharmaceutical Safety and Research) of Japan, and by the Takamatsunomiya Princess Memorial Foundation, Mitsubishi Foundation (T.S.), Naito Memorial Foundation, Uehara memorial Foundation (M.M.), and Osaka Community Foundation. Dr V. Kumar (St Louis Univ., MO) reviewed this manuscript prior to submission.

\section{References}

1. Freund J. The mode of action of immunopharmacological adjuvants. Adv Tuberc Res 1956;1:130-48 (review).

2. Seya T, Akazawa T, Uehori J, Matsumoto M, Azuma I, Toyoshima K. Role of toll-like receptors and their adaptors in adjuvant immunotherapy for cancer. Anticancer Res 2003;23:4369-76 (review).

3. Takeda K, Kaisho T, Akira S. Toll-like receptors. Annu Rev Immunol 2003;21:335-76 (review).

4. Medzhitov R, Janeway CA Jr. Innate immunity: the virtues of a nonclonal system of recognition. Cell 1997;91:295-8 (review).

5. Medzhitov R, Preston-Hurlburt P, Janeway CA. A human homologue of the Drosophila Toll protein signals activation of adaptive immunity. Nature 1997;388:394-7. 
6. Lemaitre B, Nicolas E, Michaut L, Reichhart JM, Hoffmann JA. The dorsoventral regulatory gene cassette spatzle/Toll/cactus controls the potent antifungal response in Drosophila adults. Cell 1996;86:973-81.

7. Reis e Sousa C. Toll-like receptors and dendritic cells: for whom the bug tolls. Semin Immunol 2004;16:27-34.

8. Sato A, Iwasaki A. Induction of antiviral immunity requires Toll-like receptor signaling in both stromal and dendritic cell compartments. Proc Natl Acad Sci USA 2004;101:16274-9.

9. Banchereau J, Briere F, Caux C, Davoust J, Lebecque S, Liu YJ Immunobiology of dendritic cells. Annu Rev Immunol 2000;18:767-811 (review).

10. Kaisho T, Akira S. Toll-like receptors as adjuvant receptors. Biochim Biophys Acta 2002;1589:1-13 (review)

11. Schnare M, Barton GM, Holt AC, Takeda K, Akira S, Medzhitov R. Toll-like receptors control activation of adaptive immune responses. Nat Immunol 2001;2:947-50.

12. Degli-Esposti MA, Smyth MJ. Close encounters of different kinds; dendritic cells and NK cells take centre stage. Nat Rev Immunol 2005;5: 112-24 (review).

13. Ardavin C, Amigorena S, Reis e Sousa C. Dendritic cells: immunobiology and cancer immunotherapy. Immunity 2004;20:17-23 (review).

14. Seya T, Funami K, Taniguchi M, Matsumoto M. Antibodies against human Toll-like receptors (TLRs): TLR distribution and localization in human dendritic cells. J Endotoxin Res 2005;11:369-74.

15. Inohara N, Chamaillard M, McDonald C, Nunez G. NOD-LRR proteins: role in host-microbial interactions and inflammatory disease. Апnu Rev Biochem 2004;19 [Epub ahead of print].

16. Iwasaki A, Medzhitov R. Toll-like receptor control of the adaptive immune responses. Nat Immunol 2004;10:987-94 (review).

17. Akira S. Toll-like receptor signaling. J Biol Chem 2003;278:38105-8 (review).

18. Vogel SN, Fitzgerald KA, Fenton MJ. TLRs: differential adapter utilization by toll-like receptors mediates TLR-specific patterns of gene expression. Mol Interv 2003;3:466-77 (review)

19. Seya T, Oshiumi H, Sasai M, Akazawa T, Matsumoto M. TICAM-1 and TICAM-2: toll-like receptor adapters that participate in induction of type 1 interferons. Int J Biochem Cell Biol 2005;37:524-9 (review).

20. Servant MJ, Grandvaux N, Hiscott J. Multiple signaling pathways leading to the activation of interferon regulatory factor 3. Biochem Pharmacol 2002;64:985-92 (review).

21. Meylan E, Burns K, Hofmann K, Blancheteau V, Martinon F, Kelliher M, Tschopp J. RIP1 is an essential mediator of Toll-like receptor 3-induced NF-kappa B activation. Nat Immunol 2004;5:503-7.

22. Sasai M, Matsumoto M, Seya T. The kinase complex responsible for IRF-3-mediated IFN- $\beta$ production in myeloid dendritic cells (mDC). $J$ Biochem 2005; (in press) (review)

23. Li K, Foy E, Ferreon JC, Nakamura M, Ferreon AC, Ikeda M, et al. Immune evasion by hepatitis $\mathrm{C}$ virus NS3/4A protease-mediated cleavage of the Toll-like receptor 3 adaptor protein TRIF. Proc Natl Acad Sci USA 2005;102:2992-7.

24. Barchet W, Cella M, Colonna M. Plasmacytoid dendritic cells_virus experts of innate immunity. Semin Immunol 2005;17:253-61 (review).

25. Ii M, Matsunaga N, Hazeki K, Nakamura K, Takashima K, Seya T, Hazeki O, Kitazaki T, Iizawa Y. A novel cyclohexene derivative, TAK242, selectively inhibits Toll-like receptor 4-mediated cytokine production through suppression of intracellular signaling. Mol Pharmacol. 2005; Dec 22 [Epub ahead of print].

26. Mbow ML, Sarisky RT. Modulating toll-like receptor signalling as a novel antiinfective approach. Drug News Perspect 2005;18:179-84 (review).

27. LeBouder E, Rey-Nores JE, Rushmere NK, Grigorov M, Lawn SD, Affolter M, et al. Soluble forms of Toll-like receptor (TLR)2 capable of modulating TLR2 signaling are present in human plasma and breast milk. J Immunol 2003;171:6680-9.

28. Iwami KI, Matsuguchi T, Masuda A, Kikuchi T, Musikacharoen T, Yoshikai Y. Cutting edge: naturally occurring soluble form of mouse Toll-like receptor 4 inhibits lipopolysaccharide signaling. J Immunol 2000;165:6682-6.

29. Yang E, Shin JS, Kim H, Park HW, Kim MH, Kim SJ, Choi IH. Cloning of TLR3 isoform. Yonsei Med J 2004;45:359-61.
30. Tsujita T, Tsukada H, Nakao M, Oshiumi H, Matsumoto M, Seya T. Sensing bacterial flagellin by membrane and soluble orthologs of Toll-like receptor 5 in rainbow trout (Onchorhynchus mikiss). J Biol Chem 2004;279:48588-97.

31. Tsujita T, Ishii A, Tsukada H, Matsumoto M, Che FS, Seya T. Fish soluble Toll-like receptor (TLR)5 amplifies human TLR5 response via physical binding to flagellin. Vaccine 2005; [Epub ahead of print].

32. Miyake K. Innate recognition of lipopolysaccharide by Toll-like receptor 4-MD-2. Trends Microbiol 2004;12:186-92 (review).

33. Akazawa T, Masuda H, Saeki Y, Matsumoto M, Takeda K, Tsujimura K, et al. Adjuvant-mediated tumor regression and tumor-specific cytotoxic response are impaired in MyD88-deficient mice. Cancer Res 2004;64: 757-64.

34. Uehori J, Fukase K, Akazawa T, Uematsu S, Akira S, Funami K, et al. Dendritic cell maturation induced by muramyl dipeptide (MDP) derivatives: monoacylated MDP confers TLR2/TLR4 activation. J Immunol 2005;174:7096-103

35. Matsumoto M, Funami K, Tanabe M, Oshiumi H, Shingai M, Seto Y, et al. Subcellular localization of Toll-like receptor 3 in human dendritic cells. J Immunol 2003;171:3154-62.

36. Schroeder M, Bowie AG. TLR3 in antiviral immunity: key player or bystander? Trends Immunol 2005;26:462-8 (review)

37. Gallucci S, Matzinger P. Danger signals: SOS to the immune system. Curr Opin Immunol 2001;13:114-9 (review).

38. Seong SY. Hydrophobicity: an ancient damage-associated molecular pattern that initiates innate immune responses. Nat Immunol 2004;4:469-78.

39. Dunn GP, Bruce AT, Sheenhan KC, Shankaran V, Uppaluri R, Bui JD, et al. A critical function for type I interferons in cancer immunoediting. Nat Immunol 2005;6:722-9.

40. Seya T, Matsumoto M, Akazawa T, Toyoshima K. Differential maturation of dendritic cells by TLR adapters: application to immunotherapy. Cancer Immunotherapy Ed. by K. Toyoshima, J. C. Barrett, E. Klein, Y. Hashimoto, and H. Wakasugi. Princess Takamatsu Cancer Research Fund.. Princess Takamatsu Symposia 2004; 34: 12-5.

41. Honda K, Yanai H, Takaoka A, Taniguchi T. Regulation of the type I IFN induction: a current view. Int Immunol 2005;17:1367-78 (review).

42. Pardoll DM. T cells and tumours. Nature 2001;411:1010-12 (review).

43. Zinkernagel RM. On natural and artificial vaccinations. Annu Rev Immunol 2003;21:515-546 (review).

44. Chamaillard M, Hashimoto M, Horie Y, Masumoto J, Qiu S, Saab L, et al. An essential role for NOD1 in host recognition of bacterial peptidoglycan containing diaminopimelic acid. Nat Immunol 2003;4:702-7.

45. Azuma I, Seya T. Development of immunoadjuvants for immunotherapy of cancer. Int Immunopharmacol. 2006; 1(7): 1249-59. Review.

46. Rosenberg SA, Yang JC, Restifo NP. Cancer immunotherapy: moving beyond current vaccines. Nat Med 2004;10:909-15.

47. Vermorken JB, Claessen AM, van Tinteren H, Gall HE, Ezinga R, Meijer S, et al. Active specific immunotherapy for stage II and stage III human colon cancer: a randomised trial. Lancet 1999;353: 345-50.

48. Hayashi A, Doi O, Azuma I, Toyoshima K. Immuno-friendly use of BCG-cell-wall skeleton remarkably improves the survival rate of various cancer patients. Proc Japan Acad 1998;74(Ser B):50-5.

49. Rifkin IR, Leadbetter EA, Busconi L, Viglianti G, Marshak-Rothstein A. Toll-like receptors, endogenous ligands, and systemic autoimmune disease. Immunol Rev 2005;204:27-42 (review).

50. Wille-Reece U, Flynn BJ, Lore K, Koup RA, Kedl RM, Mattapallil JJ, Weiss WR, Roederer M, Seder RA. HIV Gag protein conjugated to a Toll-like receptor $7 / 8$ agonist improves the magnitude and quality of Th1 and CD8 + T cell responses in nonhuman primates. Proc Natl Acad Sci USA 2005;102:15190-4.

51. Cuadros C, Lopez-Hernandez FJ, Dominguez AL, McClelland M, Lustgarten J. Flagellin fusion proteins as adjuvants or vaccines induce specific immune responses. Infect Immun 2004;72:2810-6.

52. Johnson GB. Activation of mammalian Toll-like receptors by endogenous agonists. Crit Rev Immunol 2003;23:15-44.

Received September 3, 2005; accepted December 26, 2005 


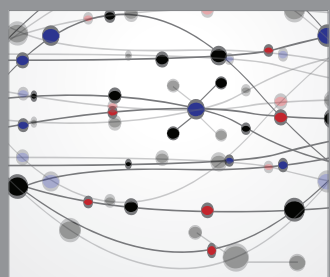

The Scientific World Journal
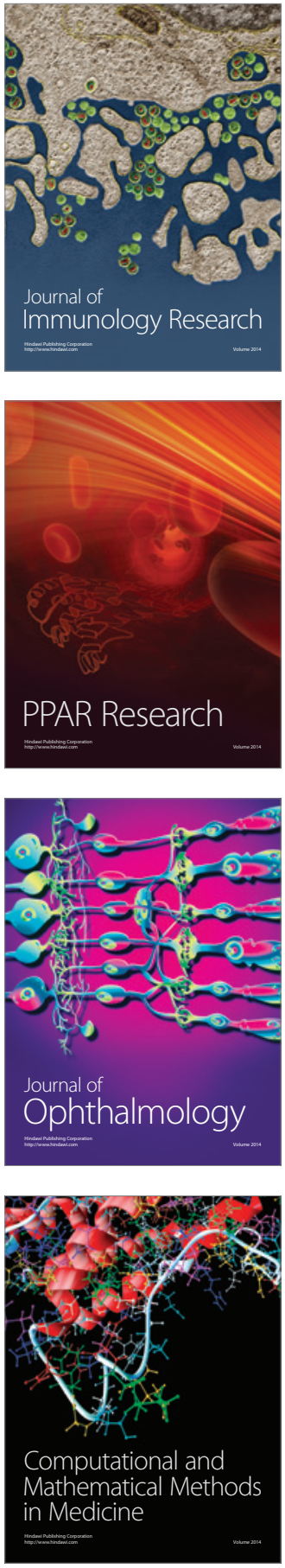

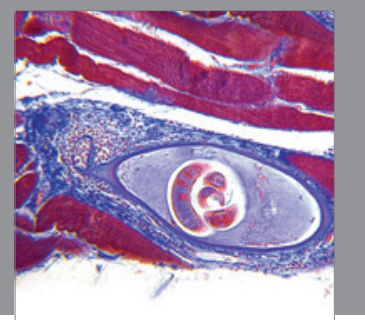

Gastroenterology

Research and Practice
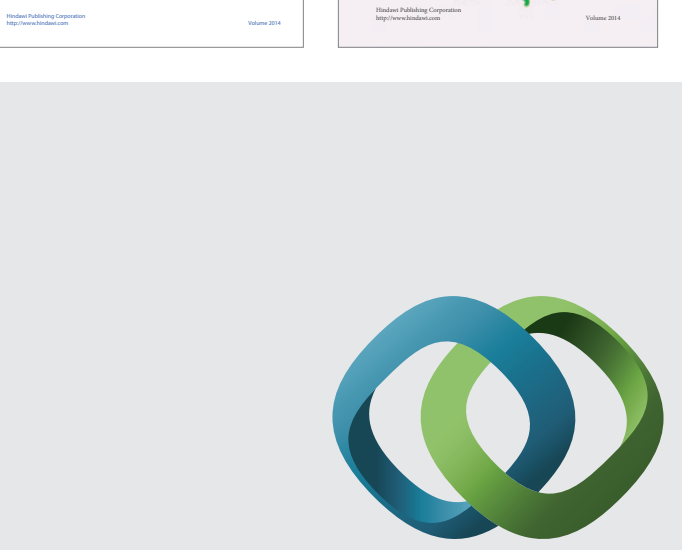

\section{Hindawi}

Submit your manuscripts at

http://www.hindawi.com
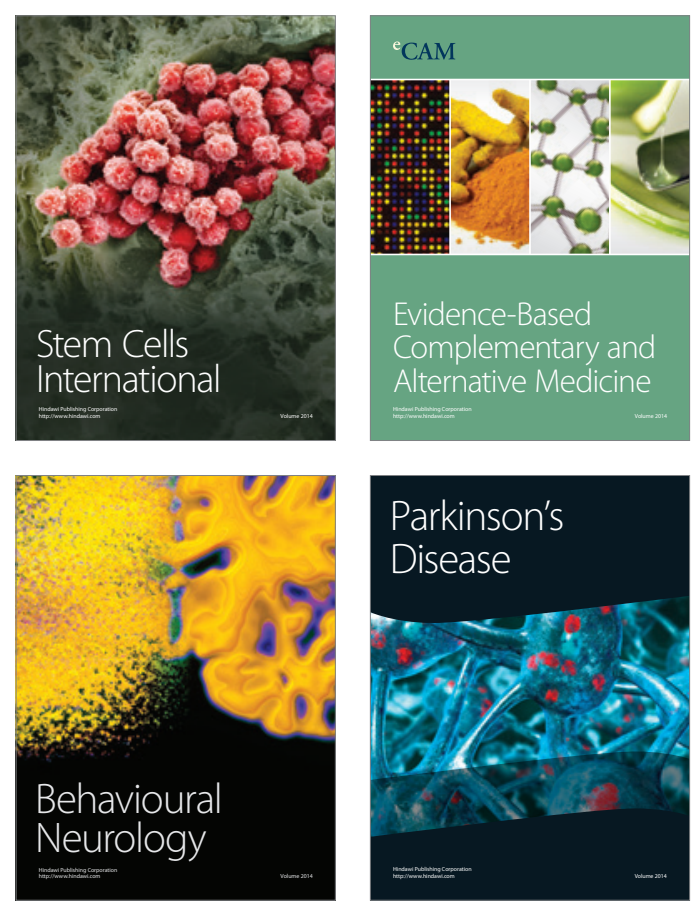

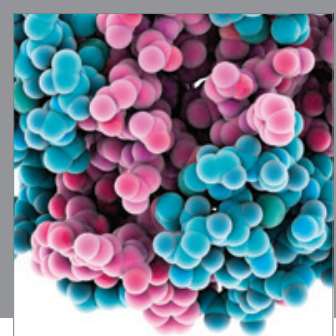

Journal of
Diabetes Research

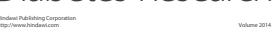

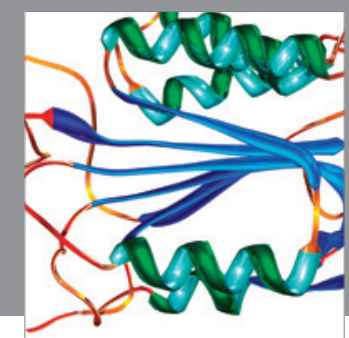

Disease Markers
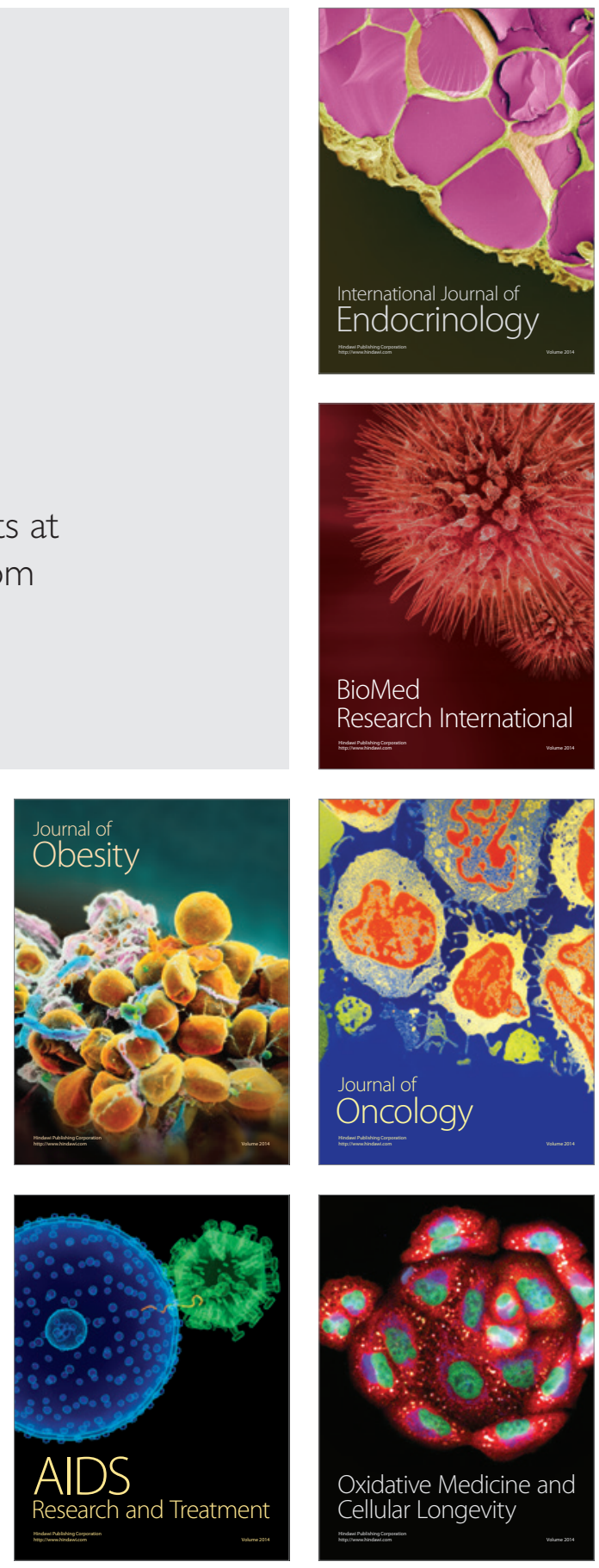